\title{
MÉTODO DE DESFOSFORAÇÃO EM PANELA PARA PRODUÇÃO DE AÇOS ULTRABAIXO CARBONO*
}

\author{
Willian Facundes ${ }^{1}$ \\ Marlon José dos Anjos Silva² \\ Túlio Carvalho da Silva Araújo ${ }^{3}$ \\ Humberto Henrique Ferreira de Assis Procópio ${ }^{4}$
}

\section{Resumo}

Algumas qualidades de aço UltraBaixo Carbono têm exigido teores de fósforo cada vez mais baixos. Quando se obtém teor de fósforo fim de sopro no convertedor acima dos níveis exigidos pelo cliente, faz-se necessário realizar um retrabalho na corrida ou promover desvios, utilizando-a em aços para outras aplicações. Para reduzir ainda mais o teor de fósforo durante o vazamento do aço do convertedor para a panela desenvolveu-se uma escória sintética desfosforante a ser utilizada quando o teor de fósforo não atende ao especificado no fim de sopro. Foram comparadas corridas com utilização da escória sintética e corridas utilizando somente cal. Obteve-se um aumento de 28,2 pontos percentuais na taxa de remoção de fósforo durante o vazamento com a utilização da escória sintética. Diante dos resultados obtidos, o produto foi aprovado para utilização na Aciaria 2 da Usiminas Ipatinga

Palavras-chave: Escória sintética; UltraBaixo Carbono; Fósforo.

\section{LADLE DEPHOSPHORIZATION METHOD TO PRODUCE ULTRALOW CARBON STEEL}

\section{Abstract}

Some qualities of UltraLow Carbon steels are requiring lower phosphorus content. When the phosphorus at the blow end inside the converter is highest then the levels required by the customer it is necessary to promote a rework or to deviate the material to another application. To further reduce the phosphorus content during the tapping of the steel from the converter to the ladle one dephosphorizer synthetic slag was developed to be used when the phosphorus content does not meet the specified at the blow end. This work compared heats with use of synthetic slag and heats using only lime. The increase in the phosphorous removal rate during tapping was 28,2 percentage points with the use of synthetic slag. Based on the results the product has been approved for use in Steelmaking 2 of Usiminas Ipatinga.

Keywords: Synthetic slag; Ultralow carbon; Phosphorus.

1 Engenheiro de Materiais, Pós-Graduação em Segurança do Trabalho, Engenheiro de Produção Pleno, Usiminas Ipatinga, MG, Brasil.

2 Engenheiro Metalurgista, Mestre em Metalurgia Extrativa, Especialista de Produção Pleno, Usiminas Ipatinga, MG, Brasil.

3 Membro da ABM, Engenheiro de Materiais, Gerente de Convertedores, Usiminas, Ipatinga, MG, Brasil.

4 Membro da ABM, Engenheiro de Materiais, Gerente de Negócios Corporativos, Vamtec Timóteo, $M G$, Brasil. 


\section{INTRODUÇÃO}

Ao longo dos anos, existe uma crescente demanda por aços ultrabaixo carbono (UBC) com baixos teores de fósforo. As indústrias consumidoras buscam melhorar as propriedades dos aços através da redução desse elemento, garantindo o desempenho do material em sua aplicação final [1]. Por outro lado, o teor de fósforo do gusa tem se elevado nos últimos anos devido ao maior teor desse elemento no minério de ferro. A média do teor de fósforo do gusa na Usiminas é de $0,121 \%$ para atender fósforo máximo especificado no aço de 0,010\% a 0,020\%, dependendo da qualidade.

Quando se obtém teor de fósforo fim de sopro no convertedor acima dos níveis exigidos pelo cliente faz-se necessário realizar um retrabalho na corrida ou promover desvios, utilizando-a em aços para outras aplicações, impactando no prazo de entrega e nos custos. A distribuição do teor de fósforo obtido ao final de sopro no convertedor LD para aços ultrabaixo carbono com especificação de baixo teor de fósforo é apresentada na Figura 1.

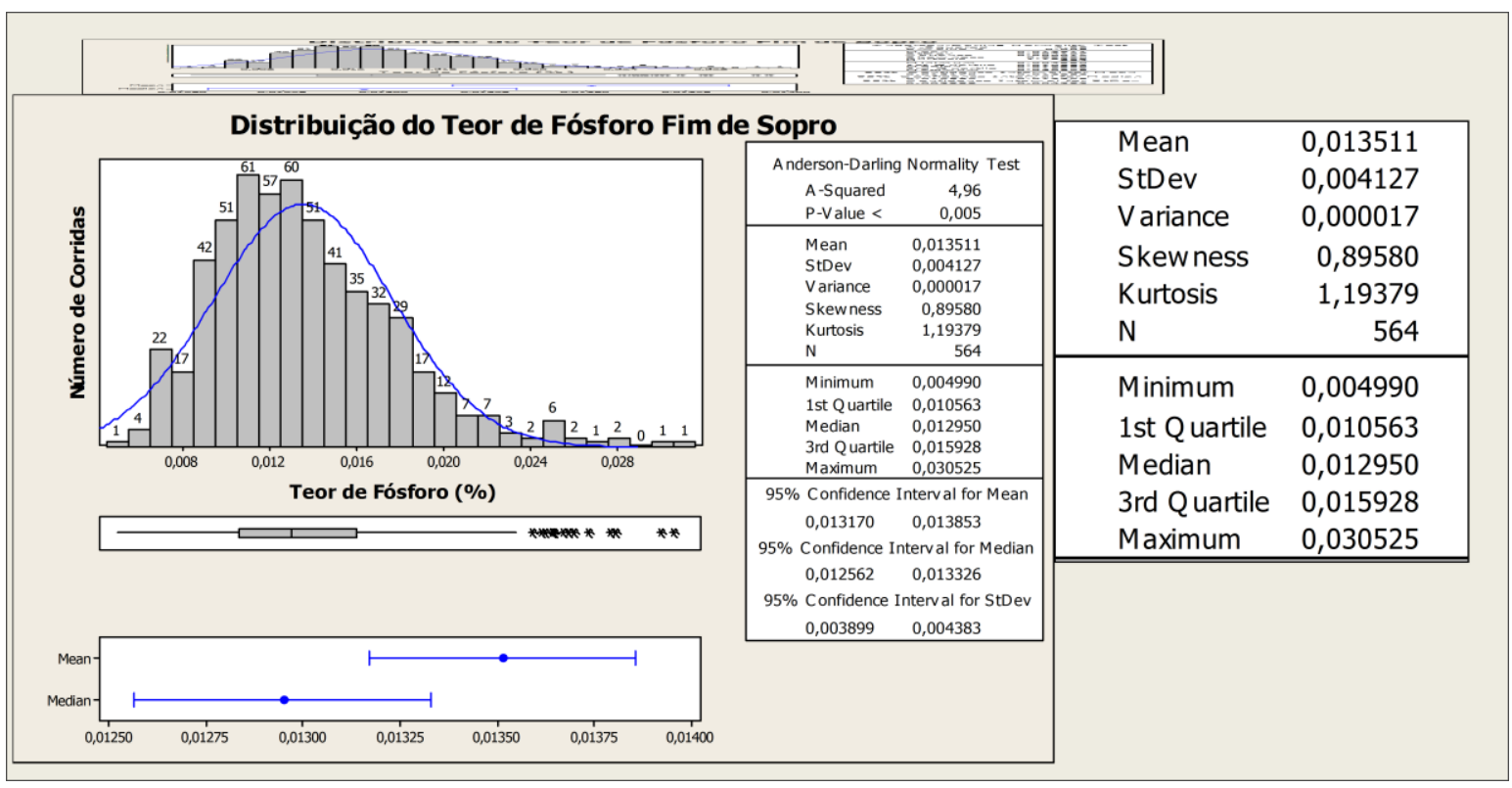

Figura 1. Distribuição do teor de fósforo no aço utrabaixo carbono.

Em uma primeira etapa, o fósforo dissolvido no banho é oxidado a $\mathrm{P}_{2} \mathrm{O}_{5}$ e, posteriormente, é incorporado a escória através da formação de um composto estável, denominado fosfato tricálcio $\left(3 \mathrm{CaO} . \mathrm{P}_{2} \mathrm{O}_{5}\right)$, responsável por reduzir a atividade do $\mathrm{P}_{2} \mathrm{O}_{5}$ [2,3]. Portanto, uma atividade de $\mathrm{CaO}$ alta aumenta a taxa de desfosforação. A oxidação do fósforo pode ser descrita através da reação 1. Júnior [2] e Gonçalves [3] descrevem a fixação do fósforo à escória, conforme descrito pela reação 2.

$$
\begin{aligned}
& 2[\mathrm{P}]+5[\mathrm{O}] \leftrightarrow\left(\mathrm{P}_{2} \mathrm{O}_{5}\right) \\
& \left(\mathrm{P}_{2} \mathrm{O}_{5}\right)+3(\mathrm{CaO}) \rightarrow\left(3 \mathrm{CaO} . \mathrm{P}_{2} \mathrm{O}_{5}\right)
\end{aligned}
$$

A eficiência da desfosforação depende também do comportamento do oxigênio e fósforo contido na escória e no aço [4], quanto maior a oxidação maior a taxa de desfosforação. Portanto, é importante que além do banho metálico, a escória 
também esteja com elevado potencial de oxigênio [5]. A Figura 2 apresenta o efeito do teor de $\mathrm{CaO}$ e FeO da escória, sobre a desfosforação.

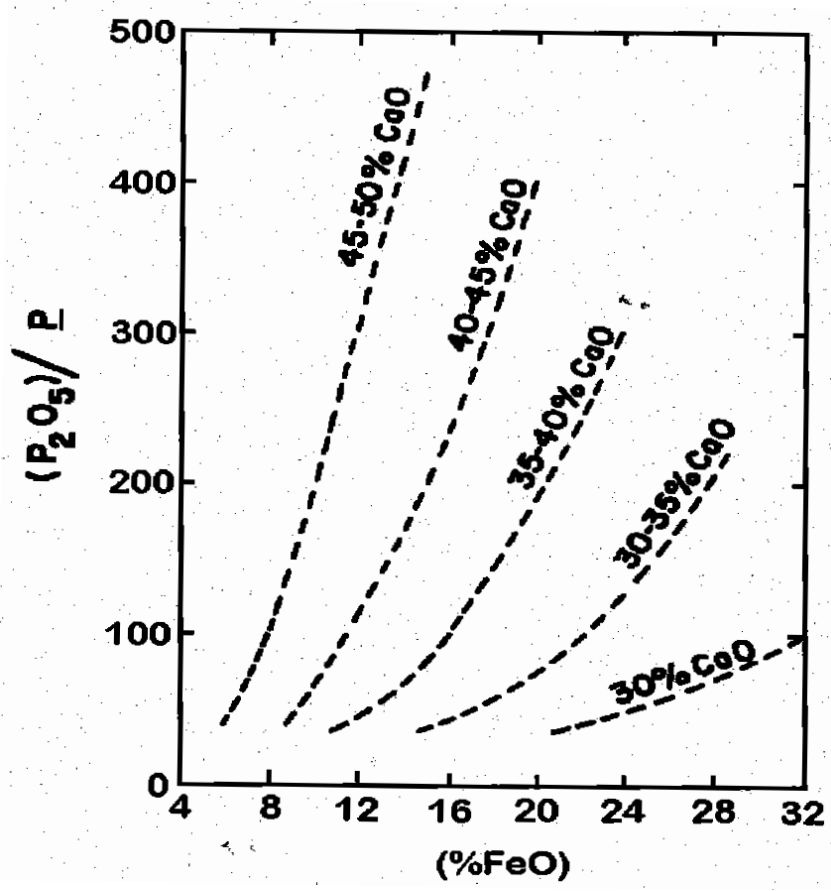

Figura 2. Efeito do teor de $\mathrm{CaO}$ e FeO da escória, sobre a desfosforação [6].

O processo de fabricação do aço ultrabaixo carbono, necessita de alto teor de oxigênio dissolvido no banho para promover a descarburação no refino secundário, esse fato facilita a utilização da prática de desfosforação em panela. A rota de produção desse aço na Usiminas é descrita na Figura 3.

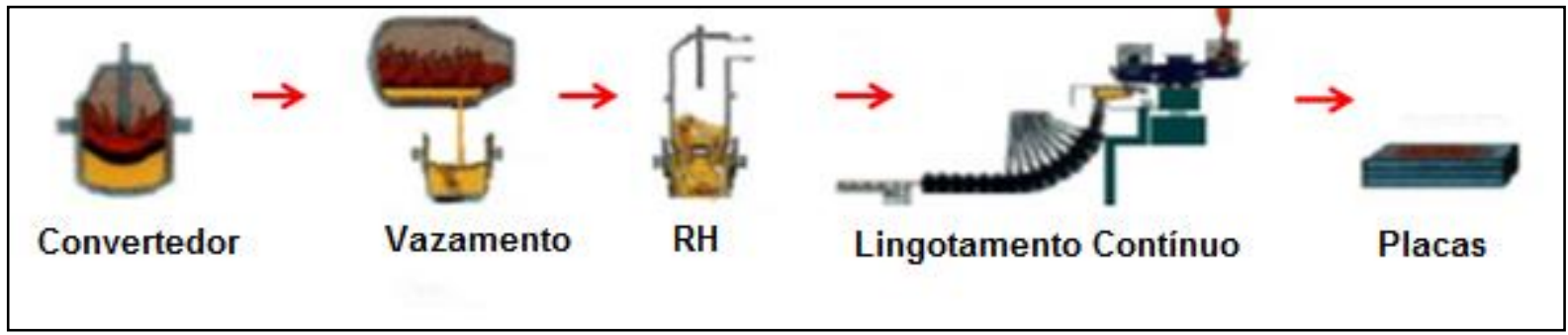

Figura 3. Fluxo de produção dos aços ultrabaixo carbono na Usiminas.

Para os casos em que ao final do sopro se obtém o teor de fósforo acima do especificado, desenvolveu-se juntamente com o fornecedor, uma escória sintética desfosforante a ser utilizada durante o vazamento do aço do convertedor para a panela.

\section{MATERIAIS E MÉTODOS}

Neste trabalho serão comparadas as taxas de remoção de fósforo durante o vazamento em corridas com utilização de uma escória sintética (cal e briquete) e corridas utilizando somente cal. Os testes foram realizados na Aciaria 2 da Usiminas Ipatinga. 
A composição química do briquete é apresentada na Tabela 1. O fluor é responsável pela redução do ponto de fusão da escória. Já o ferro, em forma de óxido de ferro, aumenta o potencial de oxigênio do sistema.

Tabela 1. Composição química do briquete desfosforante em percentual.

\begin{tabular}{ccc}
\hline Propriedades químicas & Mínimo & Máximo \\
\hline Fluor & 13,0 & 19,0 \\
\hline Ferro total & 28,0 & 38,0 \\
\hline Silício total & - & 3,5 \\
\hline
\end{tabular}

Para realização dos testes foram utilizadas 11,5 toneladas de briquete, fornecidos via bag de $500 \mathrm{~kg}$ cada. Foram acompanhadas 44 corridas, sendo 23 de teste e 21 de rotina. Para as corridas de teste utilizou-se uma mistura de 2,95 kg/t de briquete e $2,95 \mathrm{~kg} / \mathrm{t}$ de cal durante o vazamento. Para as corridas de rotina utilizou-se somente $5,90 \mathrm{~kg} / \mathrm{t}$ de cal. Para minimizar o efeito das demais variáveis como, tempo de vazamento, vida do furo e demais condições de sopro, houve alternância entre as práticas de teste e rotina durante o vazamento.

A Figura 4 apresenta a alternância de práticas durante o vazamento.
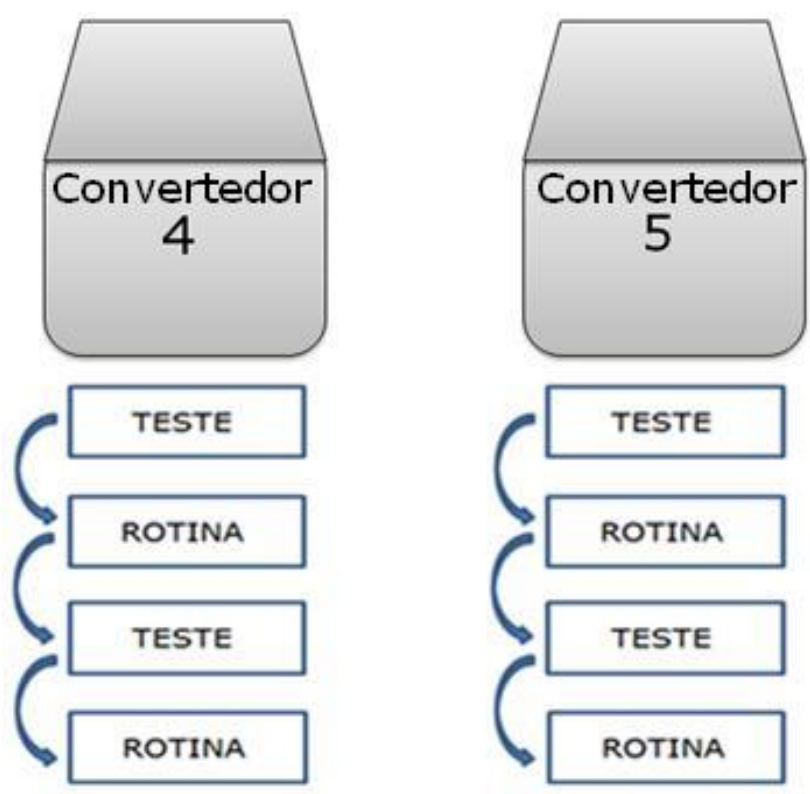

Figura 4. Alternância de práticas entre teste e rotina intercaladas durante o vazamento de aço nos convertedores 4 e 5 .

Para melhor representatividade dos resultados, alguns critérios foram adotados, sendo:

- Seleção de corridas somente com confirmação de temperatura e composição química no fim de sopro;

- Tempo de vazamento superior a 5 minutos para minimizar a passagem de escória para a panela;

- Adição da escória nos instantes iniciais de vazamento (decorridos $15 \%$ do tempo total de vazamento previsto). 
Para avaliar a remoção de fósforo pela escória, utilizou-se a Equação 3. Como o tempo de vazamento também pode influenciar na desfosforação calculou-se também a taxa de remoção de fósforo por minuto, conforme Equação 4.

$$
\begin{aligned}
& \operatorname{De}[\mathrm{P}]=\left(\frac{[\mathrm{P}] \text { inicial }-[\mathrm{P}] \text { final }}{[\mathrm{P}] \text { inicial }}\right) \times 100 \\
& \text { Taxa }[\mathrm{P}]=\left(\frac{[\mathrm{P}] \text { inicial }-[\mathrm{P}] \text { final }}{\mathrm{t}}\right)
\end{aligned}
$$

De forma que :

$[\mathrm{P}]$ inicial = Teor de fósforo inicial, ou fim de sopro, (pontos), amostra BS;

$[\mathrm{P}]$ final = Teor de fósforo obtido na panela (pontos), amostra P1;

$\mathrm{t}=$ tempo de vazamento (minutos).

\section{RESULTADOS E DISCUSSÃO}

As amostras coletadas foram analisadas no laboratório químico da Usiminas Ipatinga através de espectroscopia óptica. Observam-se na figura 5 os teores de fósforo obtidos no fim de sopro para a prática de teste e rotina. A média do teor de fósforo obitido nas corridas de teste foi de 0,0131\% e 0,0121\% para as corridas com a prática de rotina, correspondentes a 13,1 pontos e 12,1 pontos respectivamente. Com esse resultado conclui-se que não houve diferença no teor de fósforo inicial para as condições testadas.

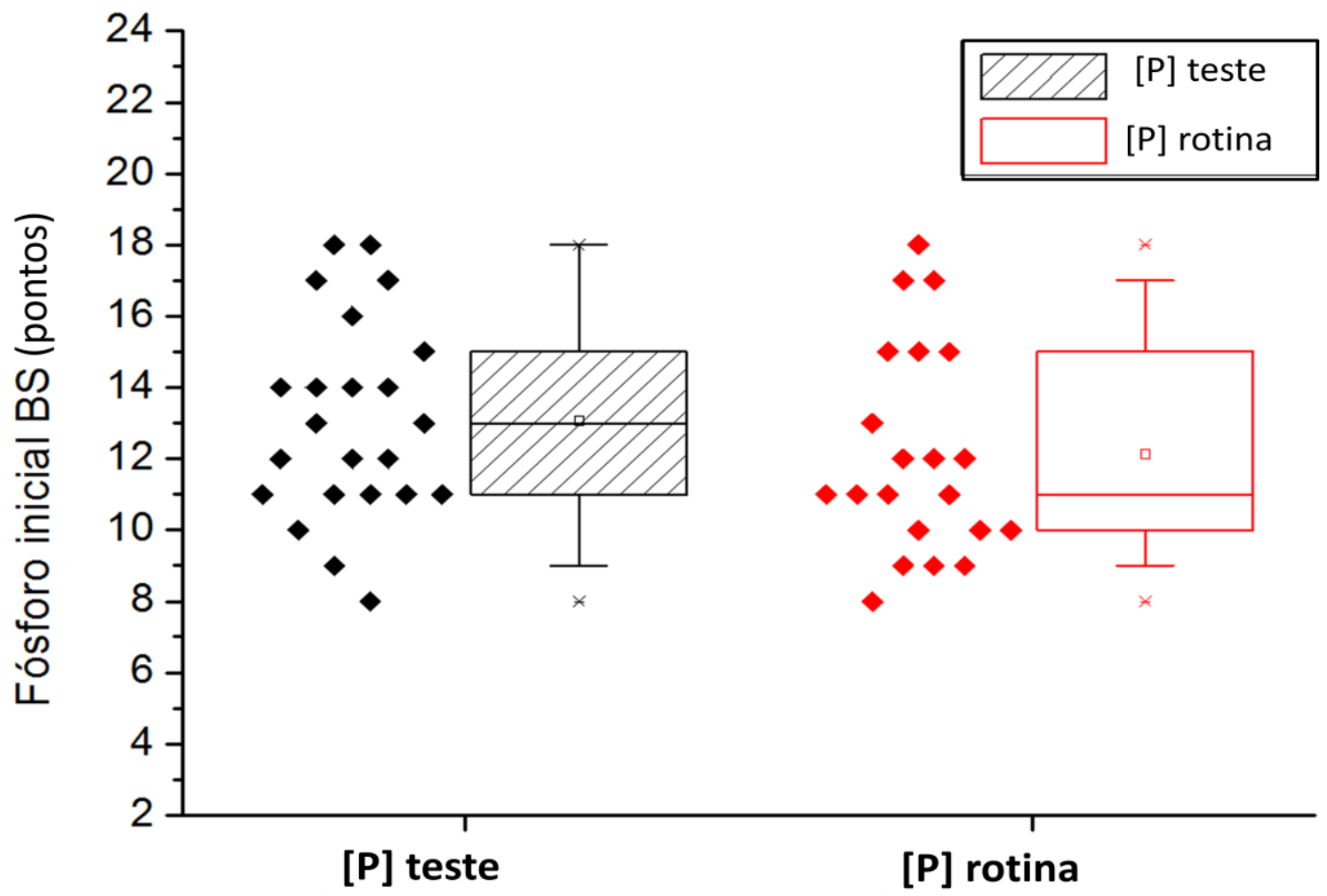

Figura 5. Teores de fósforo inicial (fim de sopro) das corridas de teste e rotina.

Como o teor inicial de fósforo pode influenciar a sua taxa de remoção, calculou-se o teste de hipótese com intervalo de confiança de $95 \%$ para checar a semelhança 
entre os resultados. O p-valor obtido é maior que 0,05 , ou seja, as médias são iguais, portanto, confirma a hipótese dos teores de fósforo iniciais serem iguais para ambos os casos.

$\mathrm{Na}$ Figura 6, são apresentados os resultados de remoção de fósforo, conforme Equação 3. Foi observada diferença do teor de fósforo obtido no fim de sopro (BS) e panela (P1). O percentual de remoção de fósforo obtido foi de $45,6 \%$ para as corridas testadas e somente $17,4 \%$ para rotina, ou seja, a taxa de desfosforação nos testes foi 28,2 pontos percentuais superior à da rotina. Em alguns casos nota-se também que não se obteve remoção de fosforo com a prática de rotina, o que não ocorreu nas corridas de teste.

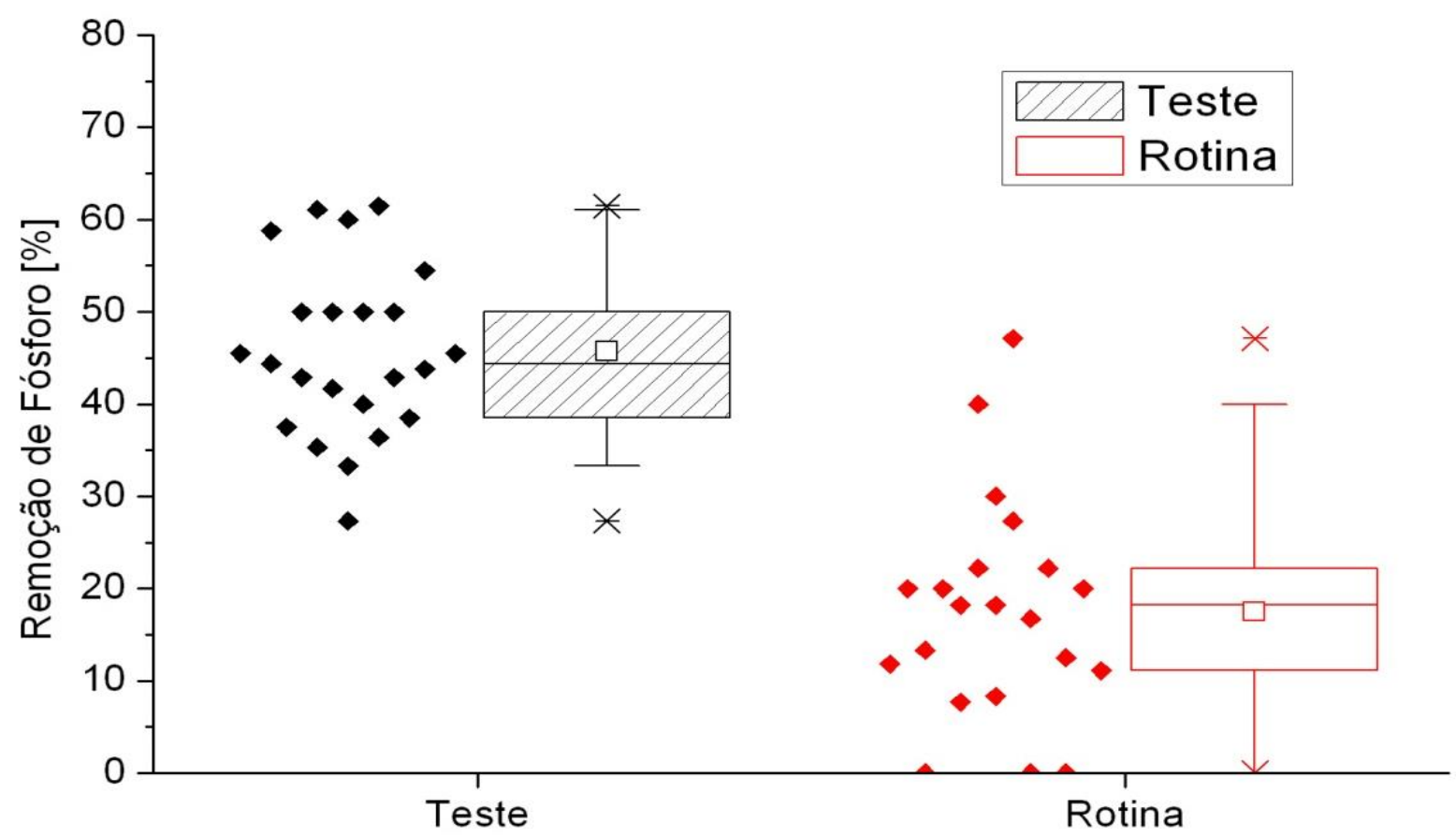

Figura 6. Percentual de remoção de fósforo com as práticas adotadas.

Realizou-se também o teste de hipótese com intervalo de confiança superior a 95\% para checar a semelhança dos resultados. O p-valor obtido foi menor que 0,05 , ou seja, as médias são diferentes; portanto, confirma a hipótese dos percentuais de remoção serem diferentes.

Calculou-se a taxa de remoção de fósforo em função do tempo de vazamento, conforme Equação 4. Nota-se que os resultados obtidos no teste são superiores aos obtidos na prática de rotina. Em média, a taxa de remoção de fósforo obtida com o teste foi de 1,1 pontos/min e com a prática de rotina de 0,3 pontos/min, conforme Figura 7. 


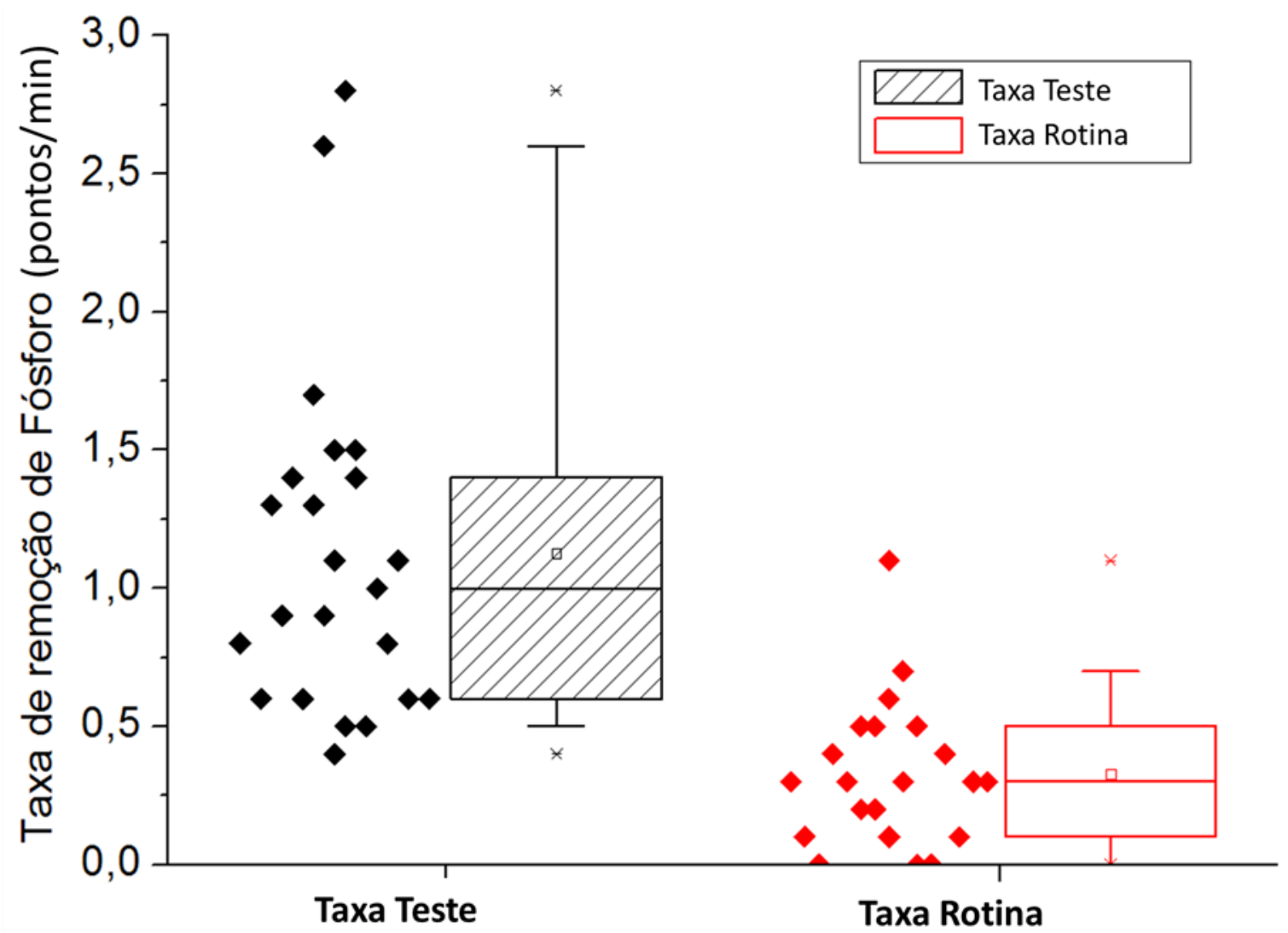

Figura 7. Taxa de remoção de fósforo em pontos/min.

\section{CONCLUSÃo}

A prática de desfosforação durante o vazamento do aço para a panela utilizando uma escória sintética contendo $2,95 \mathrm{~kg} / \mathrm{t}$ de cal e $2,95 \mathrm{~kg} / \mathrm{t}$ de briquete desfosforante apresentou remoção de fósforo 28,2 pontos percentuais superior à prática utilizando somente $5,90 \mathrm{~kg} / \mathrm{t}$ de cal. Essa prática foi aprovada tecnicamente com a finalidade de aumentar a taxa de desfosforação durante o vazamento dos aços ultrabaixo carbono em situações em que o teor de fósforo no fim de sopro encontra-se acima do especificado.

\section{REFERÊNCIAS}

1 Almeida, LP. Desenvolvimento de um Modelo de Previsão de Fósforo para o Convertedor LD da V\&M do Brasil. [Dissertação de Mestrado], UFMG, pp. 18 - 119, 2005.

2 JUNIOR, WLC et. al. Análise da Desfosforação em Convertedor na ArcelorMittal tubarão. Tecnol. Metal. Mater. Miner., São Paulo, 2013; 10(4): 346-350.

3 Gonçalves, HFP; Carneiro, CAR; Araújo, TCS. Estudo Microestrutural da Escória de Convertdeor LD e Sua Relação com o Processo de Desfosforação. Tecnol. Metal. Mater. Miner., São Paulo, 2014; 11(1): 41-49.

4 Basu, S. LAHIRI, AK, SEETHARAMAN, S. Distribution of Phosphorus and Oxygen Between Liquid Steel and Basic Oxygen Steelmaking Slag. Revue de Métallurgie. 2009. 106(I): 21-26.

5 CAMPOS, V. F. Tecnologia da Fabricação do Aço Líquido - Fundamentos; Escola de Engenharia da UFMG, 1980, 1(6): 141 - 158.

6 HERSKOVIC, J ET al. Elaboração do Aço: Fusão e Refino; ABM, São Paulo, SP, Capitulo I, Teoria da Fabricação do Aço. 